\section{(2) OPEN ACCESS}

\title{
Tapering towards DMARD-free remission in established rheumatoid arthritis: 2-year results of the TARA trial
}

\author{
Elise van Mulligen (ㄷ, ${ }^{1}$ Angelique E Weel, ${ }^{1,2,3} \mathrm{~J}$ M Hazes, ${ }^{1}$ \\ Annette van der Helm-van Mil ${ }^{0},{ }^{1,4}$ Pascal Hendrik Pieter de Jong ${ }^{1}$
}

\begin{abstract}
Handling editor Josef $S$ Smolen

- Additional material is published online only. To view please visit the journal online (http://dx.doi.org/10.1136/ annrheumdis-2020-217485).
\end{abstract}

${ }^{1}$ Rheumatology, Erasmus MC, Rotterdam, Zuid-Holland, The Netherlands

${ }^{2}$ Rheumatology, Maasstad Ziekenhuis, Rotterdam, ZuidHolland, The Netherlands ${ }^{3}$ Erasmus School of Health Policy \& Management, Erasmus Universiteit Rotterdam, Rotterdam, Zuid-Holland, The Netherlands

${ }^{4}$ Rheumatology, Leiden Universitair Medisch Centrum, Leiden, Zuid-Holland, The Netherlands

Correspondence to Ms Elise van Mulligen, Rheumatology, Erasmus MC, Rotterdam 3000 CA, The Netherlands:

elise.vanmulligen@erasmusmc. $\mathrm{nl}$

Received 6 April 2020 Revised 18 May 2020 Accepted 19 May 2020 Published Online First 1 June 2020

\section{ABSTRACT}

Objectives To evaluate the 2-year clinical effectiveness of two gradual tapering strategies. The first strategy consisted of tapering the conventional synthetic diseasemodifying antirheumatic drugs (csDMARDs) first (i.e., methotrexate in $\sim 90 \%$ ), followed by the tumour necrosis factor inhibitor (TNF-inhibitor), the second strategy consisted of tapering the TNF-inhibitor first, followed by the csDMARD.

Methods This multicentre single-blinded randomised controlled trial included patients with rheumatoid arthritis (RA) with well-controlled disease for $\geq 3$ consecutive months, defined as a Disease Activity Score (DAS) measured in 44 joints $\leq 2.4$ and a swollen joint count $\leq 1$, which was achieved with a cSDMARD and a TNF-inhibitor. Eligible patients were randomised into gradual tapering the csDMARD followed by the TNF-inhibitor, or vice versa. The primary outcome was the number of disease flares. Secondary outcomes were DMARD-free remission (DFR), DAS, functional ability (Health Assessment Questionnaire Disability Index (HAQDI)) and radiographic progression.

Results 189 patients were randomly assigned to tapering their csDMARD ( $n=94)$ or TNF-inhibitor $(n=95)$ first. The cumulative flare rate after 24 months was, respectively, $61 \%(95 \% \mathrm{Cl} 50 \%$ to $71 \%)$ and $62 \%$ (95\% Cl 52\% to 72\%). The patients who tapered their cSDMARD first were more often able to go through the entire tapering protocol and reached DFR more often than the group that tapered the TNF-inhibitor first (32\% vs $20 \%(p=0.12)$ and $21 \%$ vs $10 \%(p=0.07)$, respectively). Mean DAS and HAQ-DI over time, and radiographic progression did not differ between groups ( $p=0.45, p=0.17, p=0.8$, respectively).

Conclusion The order of tapering did not affect flare rates, DAS or HAQ-DI. DFR was achievable in $15 \%$ of patients with established RA, slightly more frequent in patients that first tapered csDMARDs. Because of similar effects from a clinical viewpoint, financial arguments may influence the decision to taper TNF-inhibitors first.

\section{Check for updates}

(C) Author(s) (or their employer(s)) 2020. Re-use permitted under CC BY-NC. No commercial re-use. See rights and permissions. Published by BMJ.

To cite: van Mulligen $\mathrm{E}$,

Weel $A E$, Hazes JM,

et al. Ann Rheum Dis

2020:79:1174-1181.

\section{INTRODUCTION}

In rheumatoid arthritis (RA) disease, outcomes have improved tremendously in the last decades, mainly due to early initiation of therapy, a treat-to-target approach and intensive therapy with conventional synthetic disease-modifying antirheumatic drugs (csDMARDs) and biologicals. As a result, remission in RA occurs more frequently. ${ }^{1}$

If patients are successfully treated and the disease is well controlled, the patient as well as the treating physician will explore the possibility to

\section{Key messages}

What is already known about this subject?

- With the possibility to taper medication, disease-modifying antirheumatic drugs (DMARD)-free remission (DFR) is suggested as a preferred ultimate goal. However, data on the ability of reaching DFR in patients with established rheumatoid arthritis (RA) are currently lacking. Also data on the best tapering strategy are limited.

\section{What does this study add?}

- The order of tapering did not affect flare rates, disease activity or physical functioning.

- DFR is achievable in $15 \%$ of patients with established RA, and therefore reachable in a minority of patients. DFR was seen slightly more frequent in patients that tapered their conventional synthetic DMARDs first.

\section{How might this impact on clinical practice or} future developments?

- Because of similar effects from a clinical viewpoint, financial arguments may influence the decision to taper tumour necrosis factor inhibitors first.

taper medication. Reasons for tapering medication are among others reduction in costs, patient preference and prevention of (long-term) side effects. Tapering treatment may, however, lead to more transient or persistent disease flares with potential harmful consequences. ${ }^{2-4}$ Previous research already showed that it is possible to taper DMARDs in RA and, therefore, current treatment recommendations advise to consider tapering therapy when patients with RA are in sustained remission. ${ }^{25}$ However, there is no consensus on the best tapering strategy.

With the possibility to taper, the final step in tapering is to fully stop DMARDs. It has been suggested that sustained DMARD-free remission (DFR, which is defined as the absence of synovitis after cessation of DMARD therapy) is a preferred ultimate outcome of RA. Previous research in early RA populations showed that $10 \%-20 \%$ of patients with RA are able to achieve this outcome, ${ }^{67}$ which was independent of the chosen treatment strategy. However, it is currently unknown if reaching DFR is a reachable outcome in established RA.

Therefore, the aim of this study is to evaluate the 2-year clinical effectiveness of two gradual tapering 


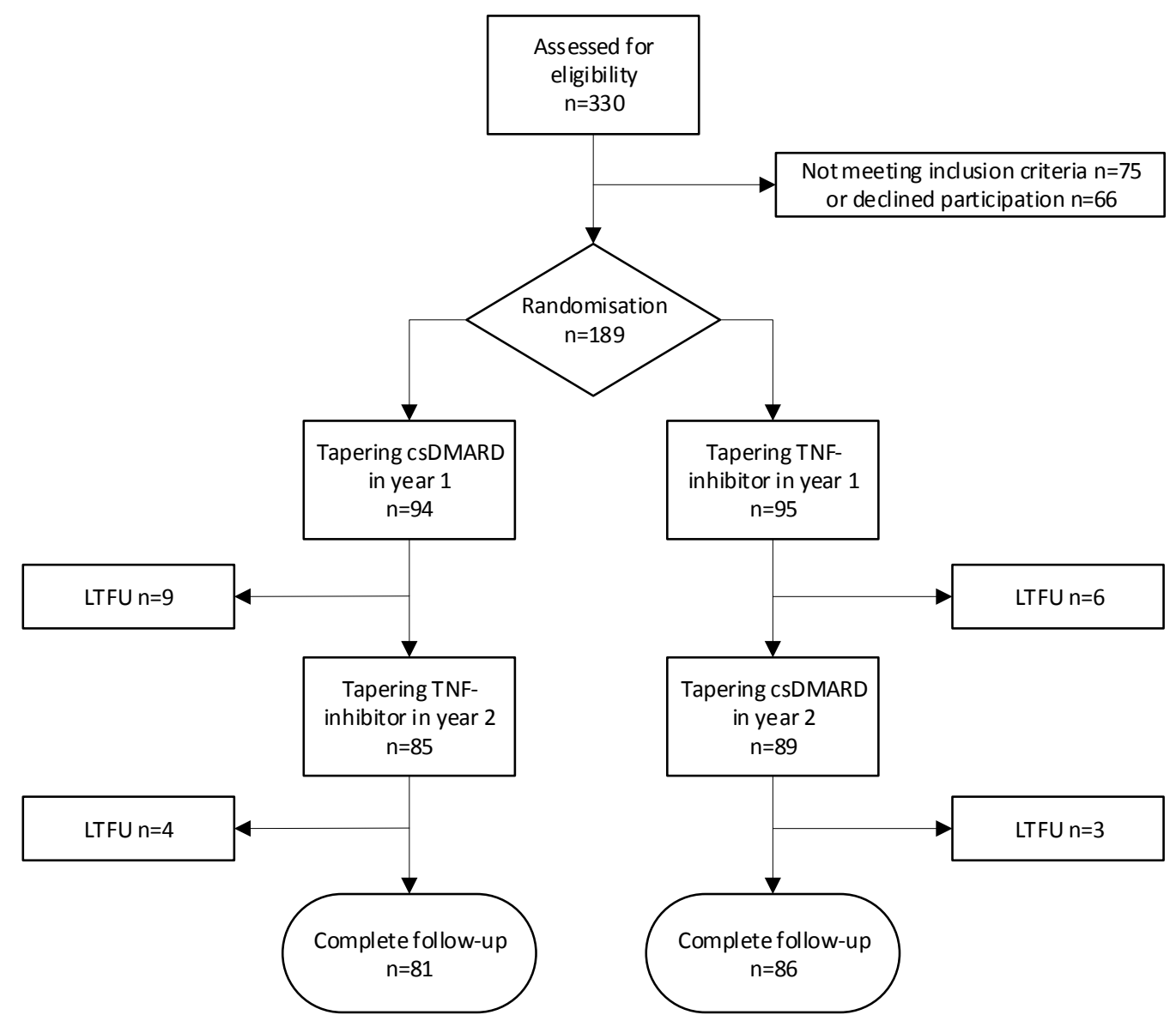

Figure 1 Flow chart of the TApering strategies in Rheumatoid Arthritis trial. Trial profile and patient participation are indicated as numbers of patients. csDMARD, conventional synthetic disease-modifying antirheumatic drug; LTFU, lost to follow-up; TNF-inhibitor, tumour necrosis factor inhibitor.

strategies, namely tapering the csDMARD first followed by the tumour necrosis factor inhibitor (TNF-inhibitor), or vice versa, in patients with established RA. We will also explore the possibility to reach DFR within this population.

\section{PATIENTS AND METHODS \\ Patient population}

Patients studied were included in the TApering strategies in Rheumatoid Arthritis (TARA) trial (NTR2754). Inclusion started September 2011 and ended July 2016. The TARA trial was a multicentre, single-blinded randomised trial, and was carried out in 12 rheumatology centres in the south-western part of the Netherlands. ${ }^{8}$ Adult patients with RA with well-controlled disease, defined as a Disease Activity Score (DAS) $\leq 2.4$ and a swollen joint count (SJC) $\leq 1$ at two consecutive time points within a 3 -month interval, using a combination of a csDMARD and TNF-inhibitor, were included. Medical ethics committees of each participating centre approved the protocol and all patients gave written informed consent before inclusion.

\section{Randomisation and blinding}

Patients were randomised using minimisation randomisation stratified for centre. Trained research nurses, blinded to the allocated tapering arm, examined patients and calculated the DAS.

\section{Tapering schedule}

Patients were randomised into tapering the csDMARD in the first year followed by tapering the TNF-inhibitor in the second year, or vice versa. The csDMARD as well as the TNF-inhibitor were gradually tapered to discontinuation in three steps. Tapering csDMARDs was realised by cutting the dosage into half, a quarter and thereafter it was stopped. TNF-inhibitors were tapered by doubling the dose interval, followed by cutting the dosage into half, and thereafter it was stopped. The total tapering schedule for each drug took 6 months, with dose adjustments every 3 months as long as there was still a well-controlled disease. At the start of the study, patients were asked to refrain from glucocorticoids (GCs). There were no restrictions on the use of non-steroidal anti-inflammatory drugs or intra-articular GC injections.

The protocol was terminated in case of a flare (DAS $>2.4$ and/or SJC>1). The previous effective dose was restarted and if necessary, medication was intensified further according to a treat-to-target approach, until low disease activity was reached again. After a flare, no further attempts were taken to taper medication during the remainder of the study.

\section{Assessments and outcomes}

Patients were examined at baseline and every 3 months thereafter. At each time point, the DAS, medication usage and selfreported questionnaires were collected, except for hand and foot radiographs, which were obtained at baseline and after 1 and 2 years of follow-up. Throughout the whole study, follow-up (serious) adverse events (AEs) were recorded.

The primary outcome was the proportion of patients with a disease flare within the entire follow-up period of 2 years. 
Table 1 Baseline characteristics of patients

\begin{tabular}{|c|c|c|}
\hline Characteristics & $\begin{array}{l}\text { Tapering csDMARD first } \\
(\mathrm{n}=94)\end{array}$ & $\begin{array}{l}\text { Tapering TNF-inhibitor } \\
\text { first }(n=95)\end{array}$ \\
\hline \multicolumn{3}{|l|}{ Demographic } \\
\hline Age (years), mean $(95 \% \mathrm{Cl})$ & 55.9 (53.0 to 58.8$)$ & 57.2 (55.0 to 59.4$)$ \\
\hline Gender, female, $\mathrm{n}(\%)$ & $67(71)$ & $58(61)$ \\
\hline \multicolumn{3}{|l|}{ Disease characteristics } \\
\hline $\begin{array}{l}\text { Symptom duration (years), } \\
\text { median (IQR) }\end{array}$ & $6.0(4.1-8.5)$ & $6.4(4.2-8.9)$ \\
\hline RF positive, $\mathrm{n}(\%)$ & $50(57)$ & $59(65)$ \\
\hline ACPA positive, $n(\%)$ & $62(71)$ & $67(75)$ \\
\hline \multicolumn{3}{|l|}{ Disease activity } \\
\hline DAS44, mean $(95 \% \mathrm{Cl})$ & $1.1(0.9$ to 1.2$)$ & $1.0(0.9$ to 1.1$)$ \\
\hline $\begin{array}{l}\text { DAS clinical remission, DAS44 } \\
<1.6, \mathrm{n}(\%)\end{array}$ & $75(80)$ & $84(88)$ \\
\hline Boolean remission, n (\%) & $31(33)$ & $35(37)$ \\
\hline HAQ-DI, mean $(95 \% \mathrm{Cl})$ & 0.52 (0.42 to 0.62$)$ & 0.47 (0.35 to 0.58$)$ \\
\hline \multicolumn{3}{|l|}{ Use of csDMARDs* } \\
\hline MTX monotherapy, n (\%) & $64(69)$ & $49(52)$ \\
\hline $\mathrm{MTX}+\mathrm{HCQ}, \mathrm{n}(\%)$ & $18(19)$ & $27(29)$ \\
\hline MTX +SASP + HCQ, n (\%) & $5(5)$ & $6(6)$ \\
\hline MTX +SASP, n (\%) & $3(3)$ & $2(2)$ \\
\hline MTX + LEF, n (\%) & $1(1)$ & $0(0)$ \\
\hline SASP monotherapy, n (\%) & $0(0)$ & $3(3)$ \\
\hline SASP +HCQ, n (\%) & $2(2)$ & $0(0)$ \\
\hline SASP + LEF, n (\%) & $0(0)$ & $1(1)$ \\
\hline LEF monotherapy, n (\%) & $1(1)$ & $3(3)$ \\
\hline $\mathrm{LEF}+\mathrm{HCQ}, \mathrm{n}(\%)$ & $0(0)$ & $1(1)$ \\
\hline HCQ monotherapy, n (\%) & $0(0)$ & $3(3)$ \\
\hline \multicolumn{3}{|l|}{ Use of TNF-inhibitor } \\
\hline Etanercept, n (\%) & $51(54)$ & $52(55)$ \\
\hline Adalimumab, n (\%) & $37(39)$ & $40(42)$ \\
\hline Certolizumab, n (\%) & $2(2)$ & $2(2)$ \\
\hline Golimumab, n (\%) & $4(4)$ & $1(1)$ \\
\hline \multicolumn{3}{|l|}{ Use of glucocorticosteroids, n (\%) } \\
\hline Oral, n (\%) & $1(1)$ & $0(0)$ \\
\hline \multicolumn{3}{|l|}{ Radiographs (hand/foot) } \\
\hline mTSS (0-488), median (IQR) & $2(0-6.5)$ & $1(0-3.5)$ \\
\hline Erosive disease, $\mathrm{n}(\%) \dagger$ & $37(39)$ & $26(27)$ \\
\hline
\end{tabular}

*Some patients used a combination of conventional synthetic disease-modifying antirheumatic drug (csDMARDs)

†Erosive disease is characterised as having $>1$ erosion in three separate joints. ACPA, anticitrullinated protein antibody; DAS44, Disease Activity Score measured in 44 joints; HAQ-DI, Health Assessment Questionnaire Disability Index; HCQ, hydroxychloroquine; LEF, leflunomide; mTSS, modified Sharp/Van der Heijde score; MTX, methotrexate; RF, rheumatoid factor; SASP, sulfasalazine; TNF, tumour necrosis factor.

Secondary endpoints were (1) the proportion of patients going through the entire tapering protocol, (2) DFR, (3) disease activity, (4) functional ability, (5) radiographic progression and (6) AEs.

Disease activity was measured with the DAS. Functional ability was measured with the Health Assessment Questionnaire Disability Index (HAQ-DI). ${ }^{9}$ Higher HAQ-DI scores indicate poorer function. Radiographic progression was measured with the modified total Sharp score (mTSS). ${ }^{10}$ Radiographs were scored chronologically by two out of three qualified assessors, who were blinded for study allocation and the identity of the patients. ${ }^{11}$ Median mTSS is reported. ${ }^{12}$ The weighted overall $\kappa$ was 0.75 with $>99 \%$ agreement. The percentage of patients with radiographic progression, defined as a change in mTSS $>0.5$ and $>1.3$ (the smallest detectable change over 2 years) is given. ${ }^{12}$ Safety monitoring took place according to Dutch guidelines, and included laboratory tests every 3 months. ${ }^{13-15}$ The medication was stopped or the dosage was lowered in case of AEs related to medication use.

\section{Statistical analysis}

The TARA trial was a superiority trial, powered to detect a $20 \%$ difference in flare rates between both tapering strategies after 1 year of follow-up, using a significance level of $\alpha=0.05$ and a power of $80 \%$, which was previously described elsewhere. ${ }^{16}$ For the current analysis, outcomes were calculated in an intentionto-treat analysis. Differences between groups in (1) cumulative flare rates, (2) proportion of patients going through the entire tapering protocol and (3) proportion of patients who reached DFR were analysed using logistic regression models. Missing data were imputed for these three analyses making use of using the last observation carried forward method. Flare-free survival was visualised with Kaplan-Meier curves, in which patients who were lost to follow-up were censored. Linear mixed models with maximum likelihood optimisation were used to compare DAS and HAQ-DI over time. Statistical comparisons of outcomes were made by Student's t-test, $\chi^{2}$ test or Wilcoxon rank-sum test when appropriate. All data were analysed using STATA V.15. A p value $\leq 0.05$ was considered statistically significant.

\section{Patient and public involvement}

Patient partners are regularly consulted as advisor for all ongoing projects in the Erasmus MC. The patient panel of the Erasmus MC consist of 15-20 patients of different age, sex and with different rheumatic diseases. Study results and study proposals are discussed on a regular basis. For the TARA study, patients were consulted for the design of the study, developing the research question and outcome parameters.

\section{RESULTS}

\section{Patients}

A total of 189 patients were randomly assigned to taper their csDMARD $(n=94)$ or TNF-inhibitor $(n=95)$ first (figure 1$)$. After 2 years of follow-up, 13 and 9 patients dropped out of the study, and complete follow-up data were obtained for 167 patients (figure 1). Patients had a median symptom duration of 6.2 years and were predominantly female (66\%) with an average age of 56.6 years (table 1). Within the group who tapered the csDMARD first, $80 \%$ had DAS remission (DAS44 $<1.6$ ), compared with $88 \%$ of patients who tapered their TNFinhibitor first. Furthermore, $33 \%$ of patients in the group who tapered the csDMARD first and 37\% of the group who tapered the TNF-inhibitor first were in Boolean remission, defined as tender joint count (TJC) $28 \leq 1$, SJC2 $8 \leq 1$, C reactive protein $(\mathrm{CRP}) \leq 10 \mathrm{mg} / \mathrm{L}$ and patient global assessment $(\mathrm{PGA}) \leq 10 \mathrm{~mm}$ (0-100 mm scale) at baseline (table 1).

\section{Primary outcome}

After 2 years of follow-up, flare rates $(95 \% \mathrm{CI})$ were $61 \%(50 \%$ to $71 \%$ ) in the group who tapered the csDMARD first, and $62 \%$ $(52 \%$ to $72 \%)$ in the group who tapered the TNF-inhibitor first $(\mathrm{p}=0.84$; figure 2$)$. The median time-to-flare (IQR) was 9.5 (6.5-21) months for patients tapering the csDMARD first, and 12 (6.5-15.5) months for patients tapering the TNF-inhibitor first. Median flare duration (IQR) was for both tapering groups 3 (3-6) months. Use of GCs was similar for both tapering arms (online supplementary table S1).

\section{DMARD-free remission}

Of the patients who tapered, respectively, their csDMARD and TNF-inhibitor first, 29 (31\%) and 20 (21\%) were able to go 
A

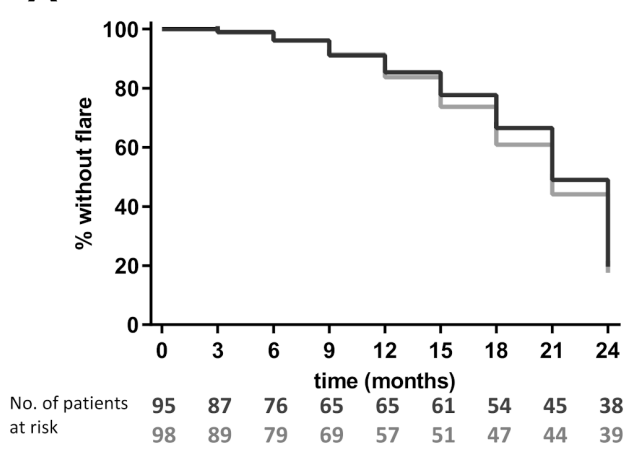

\section{C}

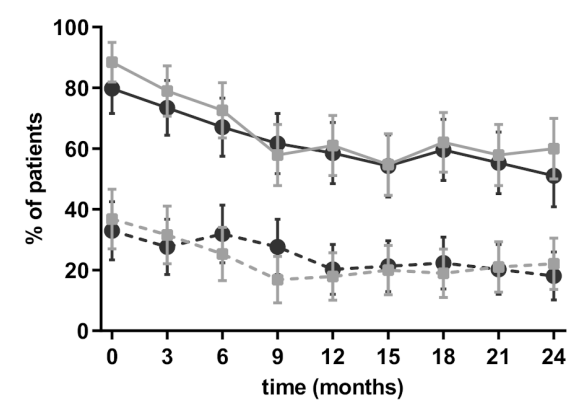

DAS $\quad \begin{array}{llllllllll}79.8 & 73.4 & 67.0 & 61.7 & 58.5 & 54.3 & 59.6 & 55.3 & 51.1\end{array}$ remission 88.4 $78.9 \quad 72.6 \quad 57.9 \quad 61.1 \quad 54.7 \quad 62.1 \quad 57.9 \quad 60.0$ $\begin{array}{llllllllll}\text { Boolean } & 33.0 & 27.7 & 31.9 & 27.7 & 20.2 & 21.3 & 22.3 & 20.2 & 18.1\end{array}$ $\begin{array}{lllllllll}\text { remission } 36.8 & 31.6 & 25.3 & 16.8 & 17.9 & 20.0 & 18.9 & 21.1 & 22.1\end{array}$

\section{Tapering csDMARD first in year 1 ,} followed by tapering TNF-inhibitor in year 2
B

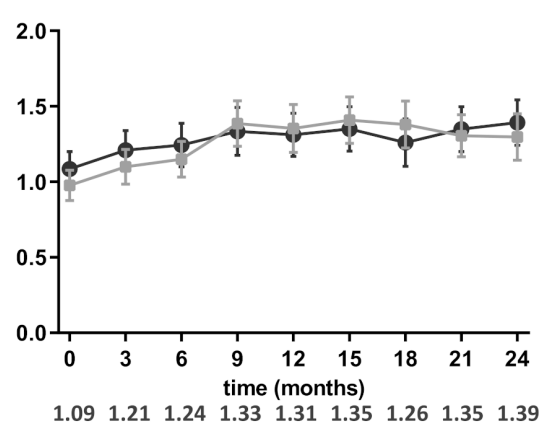

$\begin{array}{lllllllll}0.98 & 1.10 & 1.15 & 1.39 & 1.35 & 1.41 & 1.38 & 1.30 & 1.30\end{array}$

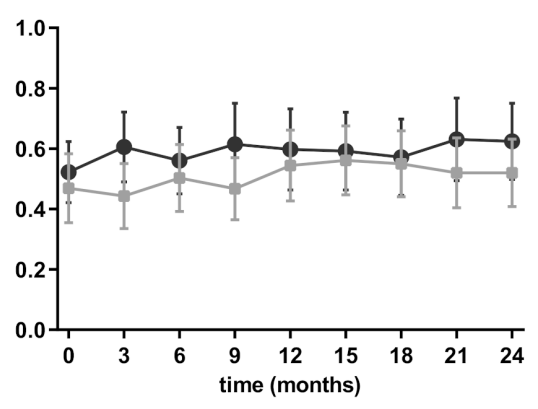

$\begin{array}{lllllllll}0.52 & 0.61 & 0.56 & 0.61 & 0.60 & 0.59 & 0.57 & 0.63 & 0.62\end{array}$ $\begin{array}{lllllllll}0.47 & 0.44 & 0.50 & 0.47 & 0.54 & 0.56 & 0.55 & 0.52 & 0.52\end{array}$

Tapering TNF-inhibitor first in year 1 ,

followed by tapering csDMARD in year 2

Figure 2 Disease activity over time. (A) Kaplan-Meier of flare-free survival, numbers below the graph indicate the number of patients at risk, (B) mean Disease Activity Score (DAS) based on 44 joints over time, (C) percentage of patients in DAS remission (DAS44 <1.6) indicated with solid lines and the percentage of patients in Boolean remission: TJC28 $\leq 1$, SJC28 $\leq 1$, C reactive protein $\leq 10 \mathrm{mg} / \mathrm{L}, \mathrm{PGA} \leq 10 \mathrm{~mm}(0-100 \mathrm{~mm}$ scale) indicated with dotted lines, (D) functional ability measured with HAQ over time. Error bars indicate $95 \%$ Cls. Numbers below graphs indicate mean values of the outcome per tapering arm, per time point, unless other indicated. csDMARD, conventional synthetic disease-modifying antirheumatic drug; DAS, Disease Activity Score; HAQ, Health Assessment Questionnaire; TNF-inhibitor, tumour necrosis factor inhibitor.

through the entire tapering protocol of tapering their TNFinhibitor and $1 \mathrm{csDMARD}(\mathrm{p}=0.12)$ (figure 3$)$. None of these patients experienced a flare after withdrawal of the csDMARD and TNF-inhibitor (period between 18 and 24 months of follow-up). Although these patients went through the entire tapering protocol, not all of them were in DFR, because some were using a combination of csDMARDs at baseline (table 1) and in the protocol only one csDMARD was tapered. This means that from the total amount of patients who tapered according to protocol, not all were in DFR. In total, 19 (20\%) patients tapering csDMARDs first and 10 (11\%) patients tapering TNF-inhibitor first were in DFR after 24 months of follow-up $(p=0.07)$. In both groups, all patients reached DFR after 18 months of follow-up, and none of them used glucocorticosteroids in the period thereafter.

\section{Disease activity, functional ability and radiographic progression}

No significant differences were found in disease activity $(p=0.45)$ and functional ability $(p=0.17)$ between both tapering groups over time (figure 2). The percentage of patients in Boolean remission after 1 year of follow-up decreased from 33\% to $20 \%$ in the group who tapered the csDMARD first and from
$37 \%$ to $18 \%$ in the group who tapered the TNF-inhibitor first, and in the second year these percentages stabilised (figure 2). Median (IQR) mTSS scores were $3(0-7.5)$ in the csDMARD and $1(0-4.5)$ in the TNF-inhibitor tapering group after 2 years of follow-up. The cumulative probability plots of both groups were similar (figure 4). Radiographic progression, defined as an mTSS increase of $>1.3$, occurred in $6.1 \%$ of the patients in the csDMARD-tapering group and $7.5 \%$ of the patients in the TNF-inhibitor tapering group $(p=0.8)$. These percentage were, respectively, $16.3 \%$ and $20 \%$ if we use an mTSS increase of $>0.5$ as definition for radiographic progression $(p=0.9)$. An increase in erosive disease $(>1$ erosion in 3 separate small hand or feet joints) after 2 years of follow-up was observed in $6.4 \%$ of patients who tapered the csDMARD first, and in $11.6 \%$ of patients who tapered their TNF-inhibitor first.

\section{Adverse events}

In total, 15 (8\%) serious AEs were reported. None of them were reported as being related to the study medication (table 2). At baseline, self-reported AEs were collected and $47.1 \%$ of all patients reported side effects. No differences were found between both tapering groups regarding the number of AEs reported and the burden of AEs (table 2). However, when 


\section{A DMARD usage over time}
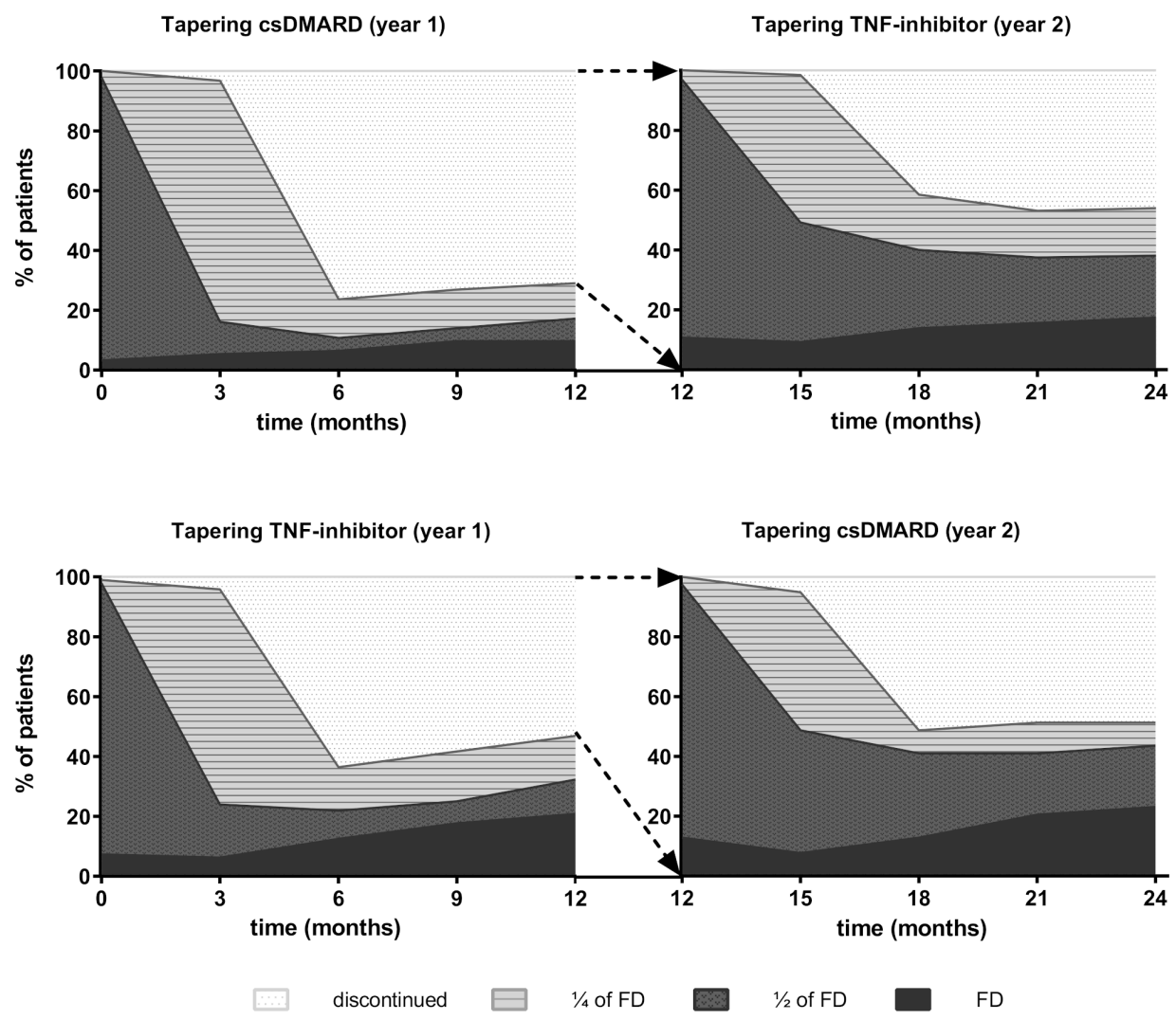

\section{B DMARD usage after 24 months}
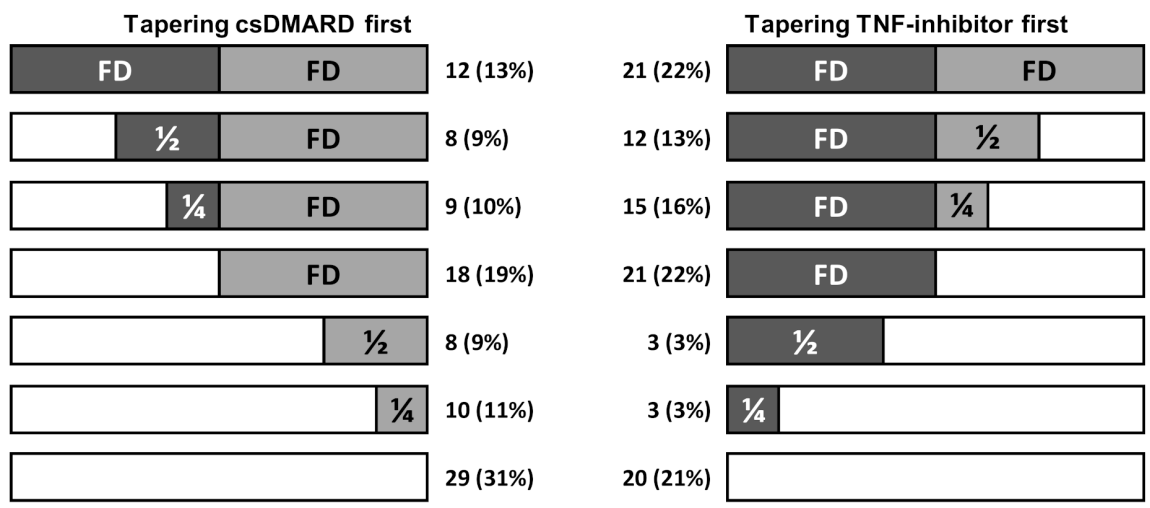

csDMARD dose

TNF-inhibitor dose

discontinued

Figure 3 Overview of medication use throughout 2 years of follow-up. In the first year, data were used of all patients; for the second year, only data were shown for patients who actually tapered their medication. When patients had a flare, it was no longer allowed to continue tapering throughout the rest of the study. (A) Disease-modifying antirheumatic drugs (DMARD) usage over time indicated for the two tapering arms, given as percentages of patients. (B) DMARD usage after 24 months. Each bar represents a certain dosage of the conventional synthetic DMARD (csDMARD) and the tumour necrosis factor inhibitor (TNF-inhibitor), ranging from no tapering on top (full dose, FD) to discontinuation of the cSDMARD and the TNFinhibitor below. Numbers (\%) next to bars indicate the number of patients who reached the indicated level of tapering after following the protocol for 24 months, as a percentage of the original TApering strategies in Rheumatoid Arthritis population.

assessing each drug separately, then AEs were more often reported for methotrexate compared with the TNF-inhibitor (35\% vs 23\%, p=0.02). The AEs related to MTX also had more impact on patients' life compared with AEs related to the TNF-inhibitor ( 20 vs $8.8, \mathrm{p}<0.0001$, measured with a Visual Analogue Scale; table 2). The self-reported AEs and their impact on patients' lives were all measured before actual tapering commenced.

\section{DISCUSSION}

In this study, the 2-year clinical effectiveness of two gradual tapering strategies in established RA was evaluated. The first 


\section{Radiological progression}

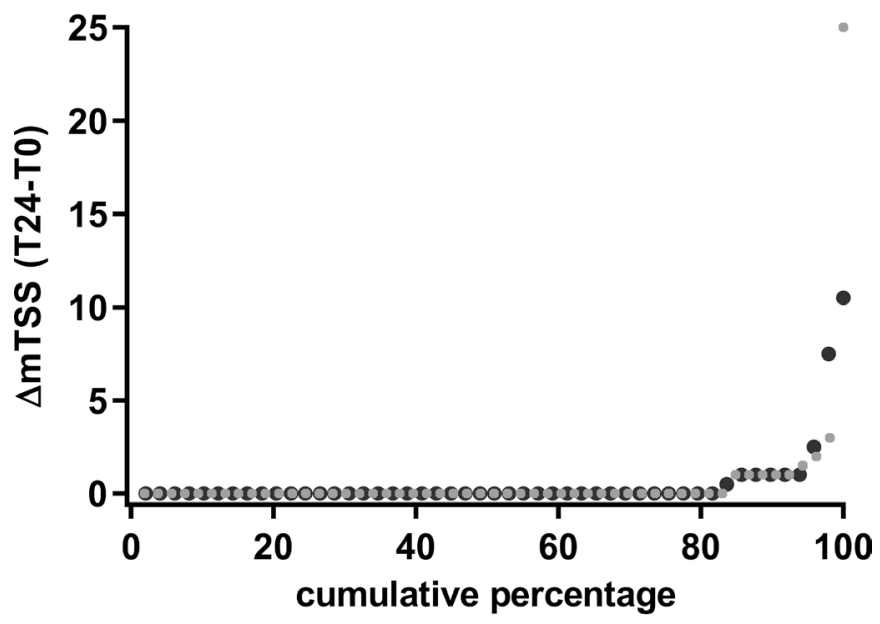

Tapering csDMARD first in year 1 , followed by tapering TNF-inhibitor in year 2

Tapering TNF-inhibitor first in year 1 , followed by tapering csDMARD in year 2

Figure 4 Radiological progression within the 2 years of follow-up. Radiological progression was measured with the modified total Sharp score (mTSS). csDMARD, conventional synthetic disease-modifying antirheumatic drug; TNF-inhibitor, tumour necrosis factor inhibitor.

strategy consisted of tapering the csDMARD first followed by the TNF-inhibitor, the second strategy consisted of tapering the TNF-inhibitor first, followed by the csDMARD. After 2 years of follow-up, $61 \%$ and $62 \%$ of patients who, respectively, tapered

\begin{tabular}{|c|c|c|}
\hline AEs & $\begin{array}{l}\text { Tapering csDMARD } \\
\text { first }(n=94)\end{array}$ & $\begin{array}{l}\text { Tapering TNF-inhibitor } \\
\text { first }(n=95)\end{array}$ \\
\hline \multicolumn{3}{|l|}{ MTX } \\
\hline Patients reporting AE due to MTX & $32(34)$ & $35(37)$ \\
\hline Off day & $7(7)$ & $8(8)$ \\
\hline Nausea & $22(23)$ & $18(19)$ \\
\hline Fatigue & $7(7)$ & $8(8)$ \\
\hline Acne & $0(0)$ & $3(3)$ \\
\hline Hair loss & $5(5)$ & $5(5)$ \\
\hline Abnormalities of oral mucosa & $1(1)$ & $3(3)$ \\
\hline Headache & $1(1)$ & $0(0)$ \\
\hline $\begin{array}{l}\text { Burden of AE due to MTX, VAS }(0-100) \text {, } \\
\text { mean (SD) }\end{array}$ & $20(27)$ & $20(27)$ \\
\hline \multicolumn{3}{|l|}{ TNF-inhibitor } \\
\hline $\begin{array}{l}\text { Patients reporting AE due to TNF- } \\
\text { inhibitor }\end{array}$ & $23(24)$ & $21(22)$ \\
\hline Pain of injection & $13(14)$ & $7(7)$ \\
\hline Fear of injection & $3(3)$ & $4(4)$ \\
\hline Irritation at place of injection & $8(9)$ & $10(11)$ \\
\hline General skin changes & $5(5)$ & $3(3)$ \\
\hline Itch & $1(1)$ & $1(1)$ \\
\hline Gastrointestinal complaints & $2(2)$ & $2(2)$ \\
\hline Fatigue & $2(2)$ & $1(1)$ \\
\hline $\begin{array}{l}\text { Burden of AE due to TNF-inhibitor, VAS } \\
(0-100) \text {, mean (SD) }\end{array}$ & $7.6(12)$ & $10(19)$ \\
\hline Serious $A E^{*}$ & $10(12)$ & $5(6)$ \\
\hline
\end{tabular}

*Serious AEs per tapering arm were, respectively: tapering conventional synthetic disease-modifying antirheumatic drug (csDMARDs) first $7 \times$ hospitalisation $(3 \times$ total hip replacement surgery, $1 \times$ pneumonia, $1 \times$ decompression shoulder, $1 \times$ pancreatitis, $1 \times$ angina pectoris), $1 \times$ herpes zoster, $1 \times$ basal cell carcinoma, $1 \times$ large-cell lung carcinoma; tapering tumour necrosis factor inhibitor (TNFinhibitor) first $4 \times$ hospitalisation $(2 \times$ peripheral vascular disease, $1 \times$ total knee replacement, $1 \times$ myocardial infarction), $1 \times$ bruised rib.

MTX, methotrexate; VAS, Visual Analogue Scale. their csDMARD or TNF-inhibitor first experienced a disease flare. Also, no differences were seen in disease activity, functional ability, radiographic progression and serious AEs. Furthermore, 31\% and $21 \%$ of patients were able to complete the entire tapering protocol. After 2 years, $20 \%$ and $11 \%$ of patients were in DFR.

The flare rates within the TARA trial were high, but within the range of previous reported flare rates $(51 \%-77 \%) .{ }^{17-20}$ Also, our median flare duration, which was 3 months, is comparable with previous tapering studies. ${ }^{3}$ This underlines the robustness of the current data and suggests that these flare rates are generalisable to clinical practice.

DFR is nowadays the closest to actual cure of RA, which might be reached by controlled tapering of medication in part of the patients. However, data on achieving DFR in patients with established RA are sparse. The Reduction of Therapy in patients with Rheumatoid arthritis in Ongoing remission (RETRO) study showed that 13 out of 27 patients with established RA (48\%) were able to reach DFR. However, these data were based on a very low sample size. ${ }^{21}$ Our DFR rate is comparable with the Leiden Early Arthritis Cohort; 158/889 (17.8\%), however, direct comparison is hampered due to various reasons, among which the difference in study design, disease stage (early vs established RA) and duration of being in DFR. ${ }^{6}$ In particular, the duration of DFR is an important measure of sustainability, and inversely related to the frequency of disease flares. ${ }^{22}$

In both tapering groups, all patients who reached DFR, reached it after 18 months of follow-up. Interestingly, none of those patients experienced a flare in the 6 months after DMARD stop, whereas other studies reported flare rates between 5\% and $25 \%$ in the first 6 months after achieving DFR. ${ }^{23-26}$ Since clearance can take more than 6 months for certain TNF-inhibitors, we might have overestimated the proportion of patients in DFR, in the group who tapered their csDMARDs first. Nonetheless, differences between groups were not significant, and we found similar flare rates in both tapering groups, which indicates that our final results are valid. Still, optimal follow-up for assessing DFR should be longer than 6 months.

A limitation of the TARA trial is that we allowed the use of $>1$ csDMARD. Because only one of the csDMARDs was tapered according to protocol, not all patients who went through the entire tapering protocol were in DFR. Ideally, we should have included only those patients who used one csDMARD combined with a TNF-inhibitor. However, subgroup analysis revealed that tapering was not more successful in patients who used multiple csDMARDs compared with the patients who used only one csDMARD.

One could argue that tapering should only take place when patients are in a 'deep' sustained remission to increase the chance at DFR and to minimise the risk of flare. Current European League Against Rheumatism (EULAR) guidelines advise to only taper medication in case of persistent remission, preferably Boolean based. ${ }^{5}$ For the TARA trial, we used a DAS $<2.4$ combined with maximum of one swollen joint, instead of the proposed remission criteria by the EULAR. This was chosen, because of a low inclusion rate. Furthermore, at time of recruitment, another trial was set up making use of the same eligibility criteria. Although we used less stringent criteria to start tapering therapy, our flare rates were comparable to other tapering strategies. Furthermore, within our study, no association was found between being in Boolean remission at baseline and staying flare-free during follow-up. This suggests that Boolean remission on its own is not a good predictor for flare-free survival when medication is tapered. Moreover, if persistent Boolean remission is the prerequisite for tapering 
therapy, fewer patients will be eligible for tapering, while in our trial only $33(17 \%)$ patients were not able to taper any treatment.

Although $15 \%$ of our patients with established RA were able to reach DFR, it is arguable whether this outweighs the risk of a disease flare (61\% in our study). Especially, since it was recently shown that disease flares have a significant effect on patients' lives, with a duration of more than 6 months. ${ }^{27} 28$

Ideally, rheumatologists want to be more certain about which patient is able to taper successfully, as current tapering strategies are based on a trial-and-error approach, which results in high flare rates that significantly influence patients' lives. Unfortunately, we still do not know which patients are more eligible for tapering and whom will have a higher chance at reaching DFR. Present data (re)confirmed that tapering treatment is possible and that DFR is achievable in a small proportion of patients even within those with an established RA. In our opinion, future studies should focus on patient subsets eligible to (continue) taper medication to reduce the amount of flares and to increase the number of patients that reach DFR.

In conclusion, the order of tapering did not affect flare rates, disease activity or physical functioning. In total, $61 \%$ of patients had a flare in the 2 years of follow-up. DFR was achievable in a small proportion of patients and was seen slightly more frequent in patients that tapered their csDMARDs first. Because of similar effects from a clinical perspective, financial arguments may influence the decision to taper TNF-inhibitors first.

Acknowledgements The authors thank the participating patients in the TARA trial for their willingness to contribute to the study and for their cooperation. They thank all rheumatologists from the following participating centres: Erasmus MC, Rotterdam; Maasstad ziekenhuis, Rotterdam; Sint Fransiscus Gasthuis \& Vlietland, Rotterdam \& Schiedam; Amphia ziekenhuis, Breda; Reinier de Graaf ziekenhuis, Delft; Antonius ziekenhuis, Utrecht; Bravis ziekenhuis, Bergen op Zoom \& Roosendaal; Groene hart ziekenhuis, Gouda; Albert Schweitzer ziekenhuis, Dordrecht; Haga ziekenhuis, Den Haag; and Beatrix ziekenhuis, Gorinchem. They also thank all studynurses, data managers, laboratory personnel, coinvestigators and others who were involved with the TARA study.

Contributors EvM, AEW, JHM and PHPdJ were responsible for acquisition of data. EvM and PHPdJ statistically analysed the data. All authors were responsible for interpretation of the data and for drafting, revising and approving the final submitted manuscript.

Funding This work was supported by ZonMw (grant number 171102014)

Disclaimer The funders of the study had no role in study design, data collection, data analysis, data interpretation, or writing of the report. The corresponding author had full access to all the data in the study and had final responsibility for the decision to submit for publication.

\section{Competing interests None declared.}

Patient and public involvement Patients and/or the public were involved in the design, or conduct, or reporting, or dissemination plans of this research. Refer to the Methods section for further details.

Patient consent for publication Not required.

Provenance and peer review Not commissioned; externally peer reviewed.

Data availability statement Data are available upon reasonable request by contacting the corresponding author.

Open access This is an open access article distributed in accordance with the Creative Commons Attribution Non Commercial (CC BY-NC 4.0) license, which permits others to distribute, remix, adapt, build upon this work non-commercially, and license their derivative works on different terms, provided the original work is properly cited, appropriate credit is given, any changes made indicated, and the use is non-commercial. See: http://creativecommons.org/licenses/by-nc/4.0/.

ORCID iDs

Elise van Mulligen http://orcid.org/0000-0003-1900-790X

Annette van der Helm-van Mil http://orcid.org/0000-0001-8572-1437

\section{REFERENCES}

1 Aletaha D, Smolen JS. Diagnosis and management of rheumatoid arthritis: a review. JAMA 2018:320:1360-72.

2 Schett G, Emery P, Tanaka Y, et al. Tapering biologic and conventional DMARD therapy in rheumatoid arthritis: current evidence and future directions. Ann Rheum Dis 2016;75:1428-37

3 Kuijper TM, Lamers-Karnebeek FBG, Jacobs JWG, et al. Flare rate in patients with rheumatoid arthritis in low disease activity or remission when tapering or stopping synthetic or biologic DMARD: a systematic review. J Rheumatol 2015;42:2012-22.

4 Henaux S, Ruyssen-Witrand A, Cantagrel A, et al. Risk of losing remission, low disease activity or radiographic progression in case of bDMARD discontinuation or tapering in rheumatoid arthritis: systematic analysis of the literature and meta-analysis. Ann Rheum Dis 2018;77:515-22

5 Smolen JS, Landewé RBM, Bijlsma JWJ, et al. EULAR recommendations for the management of rheumatoid arthritis with synthetic and biological disease-modifying antirheumatic drugs: 2019 update. Ann Rheum Dis 2020;79:685-99.

6 Ajeganova S, van Steenbergen HW, van Nies JAB, et al. Disease-modifying antirheumatic drug-free sustained remission in rheumatoid arthritis: an increasingly achievable outcome with subsidence of disease symptoms. Ann Rheum Dis 2016;75:867-73

7 Akdemir G, Heimans L, Bergstra SA, et al. Clinical and radiological outcomes of 5-year drug-free remission-steered treatment in patients with early arthritis: IMPROVED study. Ann Rheum Dis 2018;77:111-8.

8 Netherlands Trial Register(NTR). Trial NL2625 (NTR2754), 2011. Available: trialregister.nl/ trial/2625

9 Siegert CE, Vleming LJ, Vandenbroucke JP, et al. Measurement of disability in Dutch rheumatoid arthritis patients. Clin Rheumatol 1984;3:305-9.

10 van der Heijde D. How to read radiographs according to the Sharp/van der Heijde method. J Rheumatol 2000;27:261-3.

11 van Tuyl LHD, van der Heijde D, Knol DL, et al. Chronological reading of radiographs in rheumatoid arthritis increases efficiency and does not lead to bias. Ann Rheum Dis 2014;73:391-5.

12 van der Heijde D, Simon L, Smolen J, et al. How to report radiographic data in randomized clinical trials in rheumatoid arthritis: guidelines from a roundtable discussion. Arthritis Rheum 2002:47:215-8.

13 Nederlandse Vereniging voor Reumatologie. NVR Richtlijnen medicijnen: biologicals 2014: 1-26. https://www.nvr.n//wp-content/uploads/2014/11/NVR-MedicijnenUpdate_Biologicals_richtlijn-23-6-2014.pdf

14 Jul 2018Nederlandse Vereniging voor Reumatologie. NVR Richtlijnen medicijnen: sulfazalazine; 2002: 1-6. https://www.nvr.nl/wp-content/uploads/2014/11/NVRMedicijnen-richtlijn-Sulfasalazine-2002.pdf

15 Jul 2018Nederlandse Vereniging voor Reumatologie. NVR Richtlijnen medicijnen: methotrexaat; 2011: 1-6. https://www.nvr.nl/wp-content/uploads/2014/11/NVRMedicijnen-MTX-richtlijn-2009-update-2011.pdf

16 van Mulligen E, de Jong PHP, Kuijper TM, et al. Gradual tapering TNF inhibitors versus conventional synthetic DMARDs after achieving controlled disease in patients with rheumatoid arthritis: first-year results of the randomised controlled TARA study. Ann Rheum Dis 2019:78:746-53.

17 Ghiti Moghadam M, Vonkeman HE, Ten Klooster PM, et al. Stopping tumor necrosis factor inhibitor treatment in patients with established rheumatoid arthritis in remission or with stable low disease activity: a pragmatic multicenter, open-label randomized controlled trial. Arthritis Rheumatol 2016:68:1810-7.

18 van Herwaarden N, van der Maas A, Minten MJM, et al. Disease activity guided dose reduction and withdrawal of adalimumab or etanercept compared with usual care in rheumatoid arthritis: open label, randomised controlled, non-inferiority trial. BMJ 2015;350:h1389.

19 Fautrel B, Pham T, Alfaiate T, et al. Step-down strategy of spacing TNF-blocker injections for established rheumatoid arthritis in remission: results of the multicentre non-inferiority randomised open-label controlled trial (STRASS: spacing of TNF-blocker injections in rheumatoid arthritis study). Ann Rheum Dis 2016;75:59-67.

20 Smolen JS, Nash P, Durez P, et al. Maintenance, reduction, or withdrawal of etanercept after treatment with etanercept and methotrexate in patients with moderate rheumatoid arthritis (PRESERVE): a randomised controlled trial. Lancet 2013:381:918-29.

21 Haschka J, Englbrecht M, Hueber AJ, et al. Relapse rates in patients with rheumatoid arthritis in stable remission tapering or stopping antirheumatic therapy: interim results from the prospective randomised controlled RETRO study. Ann Rheum Dis 2016;75:45-51.

22 Verstappen $M$, van Mulligen $E$, de Jong PHP, et al. DMARD-free remission as novel treatment target in rheumatoid arthritis: a systematic literature review of achievability and sustainability. RMD Open 2020;6:e001220.

23 Kuijper TM, Luime JJ, de Jong PHP, et al. Tapering conventional synthetic DMARDs in patients with early arthritis in sustained remission: 2-year follow-up of the tREACH trial. Ann Rheum Dis 2016;75:2119-23.

24 Emery P, Burmester GR, Bykerk VP, et al. Evaluating drug-free remission with abatacept in early rheumatoid arthritis: results from the phase $3 \mathrm{~b}$, multicentre randomised, active-controlled AVERT study of 24 months, with a 12-month, doubleblind treatment period. Ann Rheum Dis 2015;74:19-26. 
25 Bijlsma JWJ, Welsing PMJ, Woodworth TG, et al. Early rheumatoid arthritis treated with tocilizumab, methotrexate, or their combination (U-Act-Early): a multicentre, randomised, double-blind, double-dummy, strategy trial. Lancet 2016;388:343-55.

26 Klarenbeek NB, van der Kooij SM, Güler-Yüksel M, et al. Discontinuing treatment in patients with rheumatoid arthritis in sustained clinical remission: exploratory analyses from the BeSt study. Ann Rheum Dis 2011;70:315-9.
27 Ghiti Moghadam M, Ten Klooster PM, Vonkeman HE, et al. Impact of stopping tumor necrosis factor inhibitors on rheumatoid arthritis patients' burden of disease. Arthritis Care Res 2018;70:516-24.

28 van Mulligen E, Weel AEAM, Kuijper TM, et al. The impact of a disease flare during tapering of DMARDs on the lives of rheumatoid arthritis patients. Semin Arthritis Rheum 2020;2020. doi:10.1016/j.semarthrit.2020.02.011. [Epub ahead of print: 29 Feb 2020]. 